\title{
COEFFICIENT AND STABLE IDEALS IN POLYNOMIAL RINGS
}

\author{
WiLliam HEINZER AND DAVID LANTZ
}

August 30, 1996

Let $x_{1}, \ldots, x_{d}$ be indeterminates over an infinite field $F$, let $R$ denote the polynomial ring $F\left[x_{1}, \ldots, x_{d}\right]$, and let $M$ denote the maximal ideal $\left(x_{1}, \ldots, x_{d}\right) R$. If $I$ is an $M$-primary ideal the Hilbert polynomial

$$
P_{I}(n)=e_{0}(I)\left(\begin{array}{c}
n+d-1 \\
d
\end{array}\right)-e_{1}(I)\left(\begin{array}{c}
n+d-2 \\
d-1
\end{array}\right)+\cdots+(-1)^{d} e_{d}(I)
$$

gives the length of the $R$-module $R / I^{n}$ for sufficiently large positive integers $n$. The integral closure $I^{\prime}$ of $I$ is the unique largest ideal of $R$ containing $I$ and having the same coefficient $e_{0}$ (i.e., multiplicity) as $I$, and the Ratliff-Rush ideal $\widetilde{I}$ of $I$ is the unique largest ideal containing $I$ and having the same Hilbert polynomial as I. Kishor Shah has shown in [S1] that there exists a unique chain of ideals ${ }^{1}$

$$
I \subseteq \widetilde{I}=I_{\{d\}} \subseteq \cdots \subseteq I_{\{k\}} \subseteq \cdots \subseteq I_{\{0\}}=I^{\prime}
$$

where, for $0 \leq k \leq d$, the ideal $I_{\{k\}}$ is maximal with the property of having the same coefficients $e_{0}, \ldots, e_{k}$ of its Hilbert polynomial as those of $I$. The ideal $I_{\{k\}}$ is called the $k$-th coefficient ideal of $I$. If $I=I_{\{k\}}$, we say $I$ is an $e_{k}$-ideal.

We are particularly interested in the case where $R$ is of dimension two. In this setting, an $M$-primary ideal $I$ has reduction number at most one (i.e., if $J$ is a minimal reduction of $I$, then $J I=I^{2}$ ) if and only if the Rees algebra $R[I t]$ is Cohen-Macaulay [HM, Prop. 2.6],[JV, Theorem 4.1], or [S2, Corollary 4(f)]. Moreover, the coefficients $e_{1}(I)$ and $e_{2}(I)$ are nonnegative, and it follows from $[\mathrm{Hu}$, Theorem 2.1] that $I$ has reduction number at most one if and only if $\lambda(R / I)=e_{0}(I)-e_{1}(I)$, and if this holds, then $e_{2}(I)=0$. We say that an ideal with these

\footnotetext{
${ }^{1}$ The existence of this unique chain of ideals is shown in [S1, Theorem 1] for an ideal primary for the maximal ideal of a quasi-unmixed local ring with infinite residue field. Since $R_{M}$ is regular and so, in particular, quasi-unmixed, and since the length of $R / I^{n}$ is equal to the length of $R_{M} / I^{n} R_{M}$, Shah's result also applies in our setting.
}

Typeset by $\mathcal{A M S}_{\mathcal{M}}-\mathrm{TEX}_{\mathrm{E}}$ 
properties is stable. Thus, $I$ is stable if and only if $I=\widetilde{I}$ and $e_{2}(I)=0$. Stable ideals are $e_{1^{-}}$ ideals, but it is shown in [HJL, Example 5.4] that there exist $e_{1}$-ideals $I$ for which $e_{2}(I)>0$. We are interested in a better understanding of the features of $e_{1}$-ideals and the distinguishing aspects between $e_{1}$-ideals and the more restrictive subset of stable ideals.

Our purpose in this paper is:

(1) to present examples of first coefficient and stable ideals in dimension 2,

(2) to compare the description of the coefficient ideals given by Shah in [S1, Theorems 2 and 3] with that given in [HJLS, Theorem 3.17] involving the blowup of $I$,

(3) to present examples of coefficient ideals in higher dimensions,

(4) to present two results on the existence of stable ideals in dimension 2, and to prove the $e_{1}$-closure of certain monomial ideals in dimension 2 are stable ideals.

In particular, in connection with (3) we present in more detail and with typographical corrections [HJLS, Example 3.22] that establishes the existence of examples of ideals $I$ in dimension $d$ such that for all sufficiently large positive integers $n$ one has

$$
I^{n}=\widetilde{I^{n}}=\left(I^{n}\right)_{\{d\}}<\cdots<\left(I^{n}\right)_{\{k\}}<\cdots<\left(I^{n}\right)_{\{0\}}=\left(I^{n}\right)^{\prime}
$$

and thus gives examples where all the associated coefficient ideals are distinct. Also in Section 3 we use a suggestion made to us by Karen Smith to observe that if $I$ is a monomial $M$-primary ideal, then all the associated coefficient ideals $I_{\{k\}}$ of $I$ are monomial ideals.

\section{Examples of coefficient ideals in dimension 2 .}

Let $x$ and $y$ be indeterminates over the field $F$, let $R=F[x, y]$, and let $M=(x, y) R$. The following examples illustrate the associated coefficient ideals of various ideals $I$ :

Consider the ideal $I=\left(x^{6}, x^{2} y^{4}, y^{6}\right) R$. The form ring $\mathrm{G}(I)$ of $R$ with respect to $I$ has depth one (as we have checked with Macaulay via the Rees algebra $R[I t]$ ), so $I$ and all its powers are Ratliff-Rush ideals [HLS, (1.2)]. We have the following data, including the differences $H d$ between the Hilbert function and the Hilbert polynomial as far as we have computed them:

$\begin{array}{llllll}\text { Ideal } & \text { Generators } & e_{0} & e_{1} & e_{2} & H d \\ I=\widetilde{I} & x^{6}, x^{2} y^{4}, y^{6} & 36 & 12 & 4 & {[0,0,0,0,0,0,0,0, \ldots]} \\ I_{\{1\}} & I, x^{4} y^{2} & 36 & 12 & 0 & {[0,0,0,0,0,0, \ldots]} \\ I^{\prime}=M^{6} & & 36 & 15 & 0 & {[0,0,0,0,0,0, \ldots]}\end{array}$

Since $e_{2}\left(I_{\{1\}}\right)=0$, this ideal is stable. 
To obtain a strict inclusion between $I$ and $\widetilde{I}$, as well as between the coefficient ideals, we modify as follows: Consider the ideals

$\begin{array}{llllll}\text { Ideal } & \text { Generators } & e_{0} & e_{1} & e_{2} & H d \\ I & x^{12}, x^{8} y^{4}, x^{6} y^{6}, x^{2} y^{10}, y^{12} & 144 & 60 & 4 & {[4,0,0,0,0,0, \ldots]} \\ \widetilde{I} & I, x^{4} y^{8} & 144 & 60 & 4 & {[0,0,0,0,0,0,0, \ldots]} \\ I_{\{1\}} & \widetilde{I}, x^{10} y^{2} & 144 & 60 & 0 & {[0,0,0,0,0,0, \ldots]} \\ I^{\prime}=M^{12} & & 144 & 66 & 0 & {[0,0,0,0,0,0, \ldots]}\end{array}$

Note that we have $I^{2}=(\widetilde{I})^{2}=\widetilde{\left(I^{2}\right)}$. Also, we have again that the $e_{1}$-closure $I_{\{1\}}$ of $I$ is a stable ideal.

Let us turn to an inspection of $M$-primary ideals of $R$ which have $e_{1}$-closure not stable. Since the set of stable ideals integral over a given ideal is closed under intersection [HJL, Corollary 4.4], we can speak of the stable closure of the ideal $I$, which we denote $s(I)$. Again consider the ideals:

$$
\begin{array}{llllll}
I & x^{7}, x^{5} y^{3}+x^{3} y^{5}, y^{7} & 49 & 10 & 1 & {[1,0,0,0,0,0, \ldots]} \\
\widetilde{I}=I_{\{1\}} & I, x^{6} y^{4} & 49 & 10 & 1 & {[0,0,0,0,0,0, \ldots]}
\end{array}
$$

Our verification that $I_{\{1\}}=\left(I, x^{6} y^{4}\right)$ is by using Macaulay, see [HJL, Example 5.4]. ${ }^{2}$

Another element of $(I: M)-I$ is $x^{6} y^{2}+x^{4} y^{4}+x^{2} y^{6}$, not in $\widetilde{I}$. Question: Is there a way to tell which elements of $(I: M)$ are in $\widetilde{I}$ ? In this example, $(I: M)$ is not even contained in $s(I)$, since:

$$
s(I) \quad \widetilde{I}, \text { all monomials of degree } 9 \quad 49 \quad 11 \quad 0 \quad[0,0,0,0,0,0, \ldots]
$$

It follows from Result 4.1 that this ideal is stable. To see that this is indeed the stable closure, we note that there is no intervening ideal with Hilbert coefficients $4,10,0$, since $I_{\{1\}}$ is an $e_{1}$-ideal; so the Hilbert polynomial of this ideal is the next possible for a stable ideal. (It is also true that the set of $e_{1}$-ideals integral over a given ideal is closed under intersection [HJL, Prop. 4.5].)

Another ideal having the property that its Ratliff-Rush closure is an $e_{1}$-ideal that is not stable is

$$
I \quad x^{8}, y^{8}, x^{6} y^{3}+x^{3} y^{6} \quad 64 \quad 14 \quad 1 \quad[1,0,0,0,0,0, \ldots]
$$

If we adjoin to $I$ the element $x^{7} y^{4}$, we get the same Hilbert polynomial; and we can verify that the resulting ideal and all its powers are Ratliff-Rush. Thus:

$$
\widetilde{I} \quad I, x^{7} y^{4} \quad \begin{array}{lllll}
6 & 14 & 1 & {[0,0,0,0,0,0, \ldots]}
\end{array}
$$

As in Example 5.4 of [HJL], we have shown using Macaulay that $\widetilde{I}=I_{\{1\}}$. Now we would like to

\footnotetext{
${ }^{2}$ In Example 5.4 of [HJL], $I=J$. The element $x^{6} y^{4}$ is in $(I: M)$, the preimage of the socle in $R / I$ (as is $x^{4} y^{6}$, with the same image in $R / I$.
} 
know $s(I)$; we first thought it might be

$$
\begin{array}{llllll}
K & \widetilde{I}, x^{5} y^{5} & 64 & 15 & 1 & {[0,0,0,0,0,0, \ldots]}
\end{array}
$$

But the ideal $K$ is not stable. However

$$
\begin{array}{lllll}
L & I, x^{3} y^{7}, x^{4} y^{6} & 64 & 15 & 0
\end{array} \quad[0,0,0,0,0,0, \ldots]
$$

is stable; and as in the last case, in view of the Hilbert coefficients, $L=s(I)$. In general, we would like to better understand the process of passing from an ideal to its stable closure.

\section{Passing from an ideal to its coefficient ideals.}

Let $R$ be a $d$-dimensional Noetherian quasi-unmixed local ring, and let $I$ be an ideal primary for the maximal ideal of $R$. Kishor Shah has shown that one way to attain all the coefficient ideals of $I$ is as follows: For each integer $k$ in $\{1,2, \ldots, d\}$ :

$$
I_{\{k\}}=\bigcup\left(I^{n+1}: B\right)
$$

where $n$ varies over the positive integers and $B$ varies over all the $k$-element subsets of sets of $d$ generators of minimal reductions of $I^{n}$.

In particular, if $I$ is such that $I$ and all its powers are Ratliff-Rush, i.e., G( $I)$ has positive depth, then taking a minimal reduction $\mathbf{q}$ of $I$ and considering $\left(I^{n+1}: \mathbf{q}^{n}\right)$ does not give us more than $I$, for the image of $\mathbf{q}^{n}$ in the $n$-th graded piece $I^{n} / I^{n+1}$ of $\mathrm{G}(I)$ contains a regular element of $\mathrm{G}(I)$.

Let us see how Shah's description of the coefficient ideals gives the same results as the description given in [HJLS] involving the blowup of $I$. The description of the coefficient ideals given in [HJLS, Theorem 3.17] can be phrased as follows: The ideal $I_{\{k\}}$ is the intersection of $R$ and the extensions of $I$ to the following family of overrings: $R[I / a]_{P}$, as $a$ varies over a fixed set $A$ of $d$ generators of a minimal reduction of $I$ and $P$ varies over the primes of height $\leq k$ in $R[I / a]$ that contain $I$ (or equivalently $a$ ). Let $P$ be such a prime in such a ring $R[I / a]$. Then since $R[I / a]$ is the degree- 0 piece of the localization $R[I t][1 /(a t)]$ of the Rees algebra $R[I t]$ of $I$, and this localization is a Laurent series ring in the indeterminate at over $R[I / a]$, it follows that $P R[I t][1 /(a t)] \cap R[I t]$ is a prime of height $\leq k$ in the Rees algebra. Since $a \in P$, we have $I \subseteq P$; so the image $Q$ of $P$ in the form ring $\mathrm{G}(I)=R[I t] / I R[I t]$ is a prime of height $\leq k-1$. Now for any set $C$ of $d$ generators of a minimal reduction of a power $I^{n}$ of $I, \mathrm{G}(I)$ is integral over $(R / I)[\bar{c}: c \in C]$, and the elements $\bar{c}=c t+I R[I t]$ form a regular sequence $\mathrm{G}(I)$. Thus, for any $k$-element subset $B$ of $C$, the prime $Q$ cannot contain

every $\bar{b}$ for $b \in B$ (for otherwise $\operatorname{ht}(Q) \geq k$ ). Taking preimage in the Rees algebra, there is some $b$ in $B$ for which $b t \notin P R[I t]$, so that $b / a$ is a unit in $R[I / a]_{P}$. Therefore, if $f \in\left(I^{n+1}: B\right)$, then 
$f=\left(b / a^{n}\right)^{-1}\left(b f / a^{n}\right) \in I R[I / a]_{P}$. In other words, the union of Shah's description is contained in the intersection of [HJLS].

For the reverse inclusion, given an element in the intersection of [HJLS], we must find a set $B$ of the kind described by Shah so that $\left(I^{n+1}: B\right)$ contains that element. In the Rees algebra $R[I t]$, take an irredundant primary decomposition of $I R[I t]$, say $I R[I t]=Q_{1} \cap Q_{2} \cap \cdots \cap Q_{n}$, where each $Q_{j}$ is homogeneous. For each $k=1, \ldots, d$, let $J_{k}$ denote the intersection of those $Q_{j}$ of which the radical has height at least $k+1$. Applying the "refined generalized prime avoidance lemma" $[\mathrm{S} 1$, Lemma $2(\mathrm{~F})]$ to the images $J_{k} / I R[I t]$ in $\mathrm{G}(I)$, we see that we can find, for some positive integer $N$, elements $c_{1}, \ldots, c_{d}$ in $I^{N}$ for which, for each $k=1, \ldots, d$, we have $c_{1} t^{N}, \ldots, c_{k} t^{N} \in J_{k}$ and $\operatorname{dim}\left(G(I) /\left(c_{1} t^{N}+I R[I t], \ldots, c_{k} t^{N}+I R[I t]\right) \mathrm{G}(I)\right)=d-k$. Moreover, if $J_{k-1}<J_{k}$, then we must choose $c_{n} t^{N}$ from $J_{k}-J_{k-1}$. It follows that all minimal primes of $\left(I, c_{1} t^{N}, \ldots, c_{k} t^{N}\right) R[I t]$ have height at least $k+1$. Suppose $f$ is in the intersection of [HJLS], i.e., $f$ in $R$ is also in $I R[I / a]_{P}$ for each $a$ in $A$ and each prime $P$ in $R[I / a]$ containing $I$ and of height $\leq k$. Then $f \in R[I t] \cap \bigcap\left\{I R[I t]_{P}: I \subseteq P, \operatorname{ht}(P) \leq k\right\}=\bigcap\left\{Q_{j}: \operatorname{ht}\left(\operatorname{rad}\left(Q_{j}\right)\right) \leq k\right\}$. Since $c_{1} t^{N}, \ldots, c_{k} t^{N}$ are in the remaining $Q_{j}$ (in fact, if $J_{k-1}<J_{k}$, then $c_{k} t^{N}$ has this property), we see that $f\left(c_{1} t^{N}, \ldots, c_{k} t^{N}\right.$ ) is in the degree- $N$ piece $I^{N+1} t$ of $I R[I t]$, i.e., $f \in I^{N+1}:\left(c_{1}, \ldots, c_{k}\right)$. (In fact, if $J_{k-1}<J_{k}$, then $f \in I^{N+1}: c_{k}$. Thus, the distinct coefficient ideals of $I$ are in fact colon ideals of $I^{N+1}$ by a single element of the sequence $c_{1}, \ldots, c_{d}$. The above is a very slight reworking of Shah's proof, but he does not note this realization of coefficient ideals as colons by a single element.)

\section{Coefficient ideals in higher dimensions.}

Let $R=F[x, y, z]$ where $x, y, z$ are indeterminates over an infinite field $F$, and let $M=(x, y, z) R$. Consider the ideal

$\begin{array}{lllllll}\text { Ideal } & \text { Generators } & e_{0} & e_{1} & e_{2} & e_{3} & H d \\ I & x^{6}, x^{2} y^{4}, y^{6}, x^{2} z^{4}, z^{6} & 216 & 144 & 88 & 32 & {[8,0,0,0,0,0,0, \ldots]}\end{array}$

Using Macaulay we see that the form $\operatorname{ring} \mathrm{G}(I)$ of $R$ with respect to $I$ has depth one, so $I$ and all its powers are Ratliff-Rush ideals. We claim that

$\begin{array}{lclllll}I_{\{2\}} & I, x^{4} y^{2} z^{2} & 216 & 144 & 88 & 40 & {[8,0,0,0,0,0,0, \ldots]} \\ I_{\{1\}} & \left(x^{2}, y^{2}, z^{2}\right)^{3} & 216 & 144 & 8 & 0 & {[0,0,0,0,0,0, \ldots]} \\ I^{\prime}=I_{\{0\}}=M^{6} & 216 & 180 & 20 & 0 & {[0,0,0,0,0,0, \ldots]}\end{array}$

so that

$$
I=\widetilde{I}<I_{\{2\}}<I_{\{1\}}<I^{\prime}=M^{6} .
$$


The Hilbert polynomial

$$
P_{I}(n)=e_{0}(I)\left(\begin{array}{c}
n+2 \\
3
\end{array}\right)-e_{1}(I)\left(\begin{array}{c}
n+1 \\
2
\end{array}\right)+e_{2}(I)\left(\begin{array}{c}
n \\
1
\end{array}\right)-e_{3}(I)
$$

gives the Hilbert function for all positive integers $n$, but not for $n=0$, i.e., the postulation number of $I$ is zero (as this term is used in $[\mathrm{M}]$ ).

To find these coefficient ideals of $I$ we examine the blowup of $I$. Dividing by $x^{6}$, we have the affine piece

$$
R_{x}=F\left[x, y, z,(y / x)^{4},(y / x)^{6},(z / x)^{4},(z / x)^{6}\right] \subset\left(R_{x}\right)^{\prime}=F[x, y / x, z / x] .
$$

Then $R_{x}^{(2, x)}=R_{x}\left[x^{2}(y / x)^{2}(z / x)^{2}\right]$ and $R_{x}^{(1, x)}=R_{x}\left[(y / x)^{2},(z / x)^{2}\right]$. Both of these assertions were checked by using Macaulay: The ring $R_{x}^{(2, x)}$ has depth 2 (i.e., the maximal ideal is not an associated prime of a principal ideal), and $R_{x}^{(1, x)}$ is a complete intersection.

Also, $R_{y}=F\left[x, y, z,(x / y)^{2},(x / y)^{2}(z / y)^{4},(z / y)^{6}\right]$. In this case $R_{y}=R_{y}^{(2, y)}$ (again, by Macaulay, it has depth 2), and $R_{y}^{(1)}=R_{y}\left[(z / y)^{2}\right]$ is a complete intersection. Similarly for $R_{z}$.

Thus, $I_{\{2\}}$ is the intersection of the contractions of the extensions of $I$ to $R_{x}^{(2)}, R_{y}^{(2)}$ and $R_{x}^{(2)}$. It is clear that this intersection contains $x^{4} y^{2} z^{2}$ and that the blowup of $\left(I, x^{4} y^{2} z^{2}\right) R$ has these rings as its affine pieces; checking that $\left(I, x^{4} y^{2} z^{2}\right) R$ is Ratliff-Rush, we conclude that it is $I_{\{2\}}$. Similarly for $I_{\{1\}}$.

Interlude 3.1. We thought at first that $I_{\{1\}}$ was the ideal $\left(I, x^{4} y^{2}, x^{4} z^{2}, x^{2} y^{2} z^{2}\right) R$ - we missed some of the contraction from the affine pieces of the model - so we wanted to show that this ideal is Ratliff-Rush. Eureka! After 8.5 hours of Macaulay run we obtained this verification by computing that the depth of the Rees algebra of this ideal is 3. Hence by $[\mathrm{HM}]$ the depth of the form ring with respect to this ideal is 2 .

The verification that this ideal is Ratliff-Rush using Macaulay turned out to be very time- and memory-consuming; so we were led to seek a simplifying approach. We note the following: The generators of this ideal are all in the subring $A=F\left[x^{2}, y^{2}, z^{2}\right]$ of $B=F[x, y, z]$, and $B$ is free over $A$. Let $J$ denote the $A$-ideal generated by $x^{6}, y^{6}, z^{6}, x^{2} y^{4}, x^{4} y^{2}, x^{2} z^{4}, x^{4} z^{2}, x^{2} y^{2} z^{2}$. Then the ideal above is $J B$, and $J$ is an ideal generated in degree 3 in the polynomial $\operatorname{ring} A$. Since the form ring of $B$ with respect to $J B$ is free over the form ring of $A$ with respect to $J$, if we show that the latter form ring has positive depth, then so has the former, so that all powers of $J B$ are Ratliff-Rush. Thus, we set Macaulay to computing the projective dimension of the Rees algebra of $\left(x^{3}, y^{3}, z^{3}, x y^{2}, x^{2} y, x z^{2}, x^{2} z, x y z\right) B$. But even this turned out to challenge Macaulay. 
Interlude 3.2. Another method of simplifying the computations on the defining ideal of the Rees algebra, to obtain information on the depth of the form ring and the Cohen-Macaulay property of the blowup, was shown to us by Craig Huneke. Let $I$ be a quasi-homogeneous ideal in the polynomial ring $R=F[x, y, \ldots]$; let $I=\left(f_{1}, \ldots, f_{n}\right) R$ where the $f_{i}$ 's are quasi-homogeneous. Then Macaulay can compute the kernel $J$ of the $R$-algebra epimorphism from $T=R \otimes_{F} F\left[a_{1}, \ldots, a_{n}\right]$ ( $a_{i}$ 's indeterminates) onto the Rees algebra $R[I t]$ defined by $a_{i} \mapsto f_{i} t$. The program can then find a minimal projective resolution of $J$ over $T$; the number of matrices in this resolution is the projective dimension of $R[I t]$ over $T$, so that the Auslander-Buchsbaum formula yields the depth of $R[I t]$. It follows from [HM, Theorem 2.1, page 262] that, if $G(I)$ is not Cohen-Macaulay, then the depth of $G(I)$ is one less than that of $R[I t]$. Suppose the maximal minors of the last matrix in the resolution of $J$ generate an ideal primary for $\left(x, y, \ldots, a_{1}, \ldots, a_{n}\right) T$. Then each ring in the blowup of $I$, i.e., $\operatorname{Proj}(R[I t])$, has projective dimension less than that of $R[I t]$. Thus, in this case, if $\operatorname{depth}(R[I t])=\operatorname{dim}(R)$, then $\operatorname{Proj}(R[I t])$ is Cohen-Macaulay.

But the computation of the projective resolution of $J$ may be very long and difficult, even for Macaulay. Huneke suggests passing to the quotient ring $T / x T$ of $T$ obtained by setting $x=0$. Since $x$ is regular on both $T$ and $R[I t],[\mathrm{N}, 27.2]$ the projective dimension of $J$ over $T$ is equal to the projective dimension of $J / x J$ over $T / x T$.

With regard to Example (E5) on page 387 of [HJLS], we can now confirm that the linear and constant terms of Hilbert functions of ideals between $I=\left(x^{3}, y^{3}\right)$ and its integral closure $I^{\prime}=(x, y)^{3}$ are as given there.

Let $R=F[x, y, z]$. The ideal $I=\left(x^{4}, y^{4}, z^{4}, x^{2} y^{2} z^{2}, x^{3} y^{3} z, x^{3} y z^{3}\right) R$ was pointed out to us by Les Reid to be an example of a monomial ideal that is 6 -generated but has 7 corner elements. Is this property of the ideal $I$ reflected in some way in the associated coefficient ideals of $I$ ?

Example 3.22 of [HJLS] revisited. Let $F$ be an infinite field and let $x, y_{2}, \ldots, y_{d}$ be indeterminates over $F$. Consider the affine domain $S=F\left[x,\left\{x y_{i}, y_{i}^{4}, y_{i}^{6}\right\}_{i=2}^{d}\right]$. Then

$$
S^{(1, x S)}=S^{(1)}=F\left[x,\left\{x y_{i}, y_{i}^{2}\right\}_{i=2}^{d}\right]<F\left[x,\left\{y_{i}\right\}_{i=2}^{d}\right]=S^{\prime}
$$

To see that, for $k$ in $\{1, \ldots, d-1\}$, we have $S^{(k+1, x S)}<S^{(k, x S)}$, we note that the product of $x^{2 k-2}$ and $y_{i}^{2}$, for $k$ distinct values of $i$, is an element of $S^{(k, x S)}$, since any prime $P$ in $S$ of height at most $k$ and containing $x$ does not contain $y_{i}^{4}, y_{i}^{6}$ for at least one of the $y_{i}$ 's that appear in the product, so one of the factors $y_{i}^{2}$ is a unit in $S_{P}$, and the product of the remaining factors is an element of $S$. 
But this element is not in $S^{(k+1, x S)}$ because it is not in the localization of $S$ at the prime ideal of height $k+1$ generated by $x$ and $x y_{i}, y_{i}^{4}, y_{i}^{6}$ for the $y_{i}$ 's that appear in the product.

The domain $S$ is an affine piece of the blowup of the ideal $I$ generated by $x^{6}, x^{2}\left(x y_{i}\right)^{4},\left(x y_{i}\right)^{6}$, $i=2, \ldots, d$ in the polynomial ring $R=F\left[x,\left\{x y_{i}\right\}_{i=2}^{d}\right]$. Forming the rings of fractions of $R, S$ with respect to the complement in $R$ of the maximal ideal $M=\left(x,\left\{x y_{i}\right\}_{i=2}^{d}\right) R$ yields a regular local ring $R_{M}$, and $(R-M)^{-1} S$ is an affine piece of the blowup of $I R_{M}$ which retains the properties verified in the last paragraph.

Moreover, since the extensions of the affine piece of the blowup of $I$ as described in [HJLS] are distinct, it follows that, for sufficiently high powers of $I$, the contractions of these powers from the various extensions of the blowup are distinct; i.e., for $n$ sufficiently large,

$$
\widetilde{\left(I^{n}\right)}=\left(I^{n}\right)_{\{d\}}<\left(I^{n}\right)_{\{d-1\}}<\cdots<\left(I^{n}\right)_{\{0\}}=\left(I^{n}\right)^{\prime} \text {. }
$$

In fact, we believe that these strict inclusions hold for $n=1$.

Observation 3.3. Let $R=F\left[x_{1}, \ldots, x_{d}\right]$ be a polynomial ring in $d$ variables $x_{1}, \ldots, x_{d}$ over an infinite field $F$, let $M=\left(x_{1}, \ldots, x_{d}\right) R$ and let $I$ be an $M$-primary ideal. We say that $I$ is a monomial ideal if $I$ is generated by monomials in $x_{1}, \ldots, x_{d}$. Karen Smith suggested to us the following argument to show that if $I$ is a monomial ideal, then all the associated coefficient ideals $I_{\{k\}}$ of $I$ are also monomial ideals. For each $d$-tuple $a=\left(a_{1}, \ldots, a_{d}\right)$ in the algebraic $d$-torus $F^{*} \times \cdots \times F^{*}$, where $F^{*}=F-0$ is the multiplicative group of units of $F$, define an $F$-automorphism $\phi_{a}: R \rightarrow R$ by setting $\phi_{a}\left(x_{i}\right)=a_{i} x_{i}$ for $1 \leq i \leq d$. To show $I_{\{k\}}$ is a monomial ideal it suffices to show $\phi_{a}\left(I_{\{k\}}\right)=I_{\{k\}}$ for each $a=\left(a_{1}, \ldots, a_{d}\right)$. By assumption $I=\left(f_{1}, \ldots, f_{n}\right) R$, where each $f_{i}$ is a nonzero monomial. Let $R_{i}=R\left[f_{1} / f_{i}, \ldots, f_{n} / f_{i}\right]$. Then $\phi_{a}$ naturally extends to an $F$-automorphism of $R_{i}$ which we continue to call $\phi_{a}$, and $I R_{i}=f_{i} R_{i}$ is mapped to itself under $\phi_{a}$. The invariance of $f_{i} R_{i}$ under $\phi_{a}$ implies that the union of the associated primes of $f_{i} R_{i}$ of height at most $k$ in $R_{i}$ is mapped onto itself under $\phi_{a}$. Therefore $\phi_{a}$ extends to an automorphism of the localization $T_{i k}$ of $R_{i}$ at the complement of the union of the associated primes of $f_{i} R_{i}$ of height at most $k$. It follows that $f_{i} T_{i k} \cap R$ is mapped onto itself by $\phi_{a}$. By [HJLS, Theorem 3.17], $I_{\{k\}}=\cap_{i=1}^{n} f_{i} T_{i k} \cap R$. Therefore the ideal $I_{\{k\}}$ is mapped onto itself by $\phi_{a}$, so $I_{\{k\}}$ is a monomial ideal.

\section{Stable ideals in dimension 2 .}

Let us examine with $R=F[x, y]$ what ideals between $I=\left(x^{n}, y^{n}\right)$ and its integral closure $I^{\prime}=(x, y)^{n} R$ are stable. The following two results show that many of these ideals are stable: 
Result 4.1. Let $a, b$ be a minimal reduction of the height- 2 ideal $A$ in the $\operatorname{ring} R$, and $n$ be a positive integer; set $I=\left(a^{n}, b^{n}\right)$. Then $I$ is a minimal reduction of $A^{n}$; suppose the reduction number is 1 , i.e., $I A^{n}=A^{2 n}$. Then for every nonnegative integer $j$ we have $I A^{n+j}=A^{2 n+j}$. Set $J=I+A^{n+i+1}$ for a positive integer $i$; then we have

$$
J^{2}=I J+\left(A^{n+i+1}\right)^{2}=I J+A^{2 n+2 i+2}=I J+I A^{n+2 i+2} \subseteq I\left(J+A^{n+i+1}\right)=I J ;
$$

i.e., $J$ is stable. Moreover, for each ideal $K \subseteq A^{n+i}$ the ideal $L=J+K$ is also stable. For, we have

$$
\begin{aligned}
L^{2}=J^{2}+J K+K^{2} & \subseteq I J+\left(I+A^{n+i+1}\right) K+A^{2 n+2 i} \\
& \subseteq I(J+K)+A^{2 n+2 i+1}+A^{2 n+2 i}=I(J+K)+A^{2 n+2 i} \\
& =I\left(J+K+A^{n+2 i}\right)=I(J+K)=I L
\end{aligned}
$$

where we have used $A^{n+2 i} \subseteq J$.

Result 4.2. Again, let $R$ be a ring. Let $a, b$ be a $R$-sequence, and $m, n$ be positive integers; and set $I=\left(a^{m}, b^{n}\right)$. Then for any ideal $J$ contained in $a^{\lceil m / 2\rceil} b^{\lceil n / 2\rceil} R$, the sum $I+J$ is stable. For, $\left(a^{\lceil m / 2\rceil} b^{\lceil n / 2\rceil}\right)^{2} \in I^{2}$, so $J^{2} \subseteq I^{2}$, so $(I+J)^{2}=I^{2}+I J+J^{2} \subseteq I^{2}+I J=I(I+J)$.

Applying these paragraphs to $x, y$ in $F[x, y]$, we see that: (1) for any integer $n \geq 2$, we can find stable ideals $I$ between $\left(x^{n}, y^{n}\right) R$ and $(x, y)^{n} R$ such that $e_{0}(I)=n^{2}$ and $e_{2}(I)=0$ (both necessarily) and $e_{1}(I)$ is any integer from 0 to $(n-2)(n-1) / 2$; and (2) for any positive integers $m, n$, we can find stable ideals $I$ between $\left(x^{m}, y^{n}\right) R$ and its integral closure with $e_{0}(I)=m n$, $e_{2}(I)=0$ (again, both necessarily) and $e_{1}(I)$ is any integer from 0 to $\left\lceil\frac{m}{2}\right\rceil\left\lceil\frac{n}{2}\right\rceil$.

In (4.3) we prove that the $e_{1}$-closure of certain monomial ideals are stable. This shows that examples such as [HJL, Example 5.4] are of necessity not generated by monomials.

Observation 4.3. Let $R=F[x, y]$ where $x$ and $y$ are indeterminates over the infinite field $F$, let $m$ and $n$ be positive integers and suppose $I$ is a monomial ideal of $R$ integral over $\left(x^{m}, y^{n}\right) R$. We show that the $e_{1}$-closure $J$ of $I$ is stable. By [HJLS, Theorem 3.17] (see also [HJL, (3.2)], $J=I D \cap R$, where $D$ is the first coefficient domain of $I$. Then $D$ is also the first coefficient domain of $J$ and each of the rings $R\left[J / x^{m}\right]$ and $R\left[J / y^{n}\right]$ is contained in $D$. Therefore $J R\left[J^{2} / x^{m} y^{n}\right] \cap R=J$. It follows that $\left(J^{3}: x^{m} y^{n}\right)=J$. To show that $J$ is stable, it suffices to show that $J^{2} \subseteq\left(x^{m}, y^{n}\right) J$. The Briancon-Skoda Theorem [LS, Theorem 1] implies $J^{2} \subseteq\left(x^{m}, y^{n}\right) R$. By (3.3), the ideal $J$ is 
a monomial ideal. Let $a \in J^{2}$ with $a$ a monomial. Then $a \in\left(x^{m}, y^{n}\right) R$ implies either $a \in x^{m} R$ or $a \in y^{n} R$. Suppose $a=x^{m} b$ with $b \in R$. Then $b \in\left(J^{2}: x^{m}\right) \subseteq\left(J^{3}: x^{m} y^{n}\right)=J$ which means $a \in\left(x^{m}, y^{n}\right) J$. A similar arguement applies in case $a \in y^{n} R$. Therefore $J$ is stable.

\section{REFERENCES}

[HJL] W. Heinzer, B. Johnston and D. Lantz, First coefficient domains and ideals of reduction number one, Comm. Algebra 21 (1993), 3797-3827.

[HJLS] W. Heinzer, B. Johnston, D. Lantz and K. Shah, Coefficient ideals in and blowups of a commutative Noetherian domain, J. Algebra 162 (1993), 355-391.

[HLS] W. Heinzer, D. Lantz and K. Shah, The Ratliff-Rush ideals in a Noetherian ring, Comm. Algebra 20 (1992), 591-622.

[HM] S. Huckaba and T. Marley, Depth properties of Rees algebras and associated graded rings, J. Algebra 156 (1993), 259-271.

[Hu] C. Huneke, Hilbert functions and symbolic powers, Michigan Math. J. 34 (1987), 293-318.

$[\mathrm{J}] \quad$ B. Johnston, Toward parametric Cohen-Macaulifications of 2-dimensional finite local cohomology domains, Math. Z. 207 (1991), 569-581.

[JV] B. Johnston and J. Verma, On the length formula of Hoskin and Deligne and associated graded rings of two-dimensional regular local rings, Math. Proc. Camb. Phil. Soc. 111 (1992), 423-432.

[LS] J. Lipman and A. Sathaye, Jacobian ideals and a theorem of Briancon-Skoda, Michigan Math. J. 28 (1981), 199-222.

[M] T. Marley, The coefficients of the Hilbert polynomial and the reduction number of an ideal, J. London Math. Soc. (2) 156 (1989), 1-8.

[N] M. Nagata, Local Rings, Interscience, New York, 1962.

[S1] K. Shah, Coefficient ideals, Trans. Amer. Math. Soc. 327 (1991), 373-384.

[S2] K. Shah, On the Cohen-Macaulayness of the fiber cone of an ideal, J. Algebra 143 (1991), 156-172. 